\title{
Méthodologie de l'amélioration du blé tendre (Triticum aestivum L). II. Étude et analyse de deux premiers cycles de sélection récurrente
}

\author{
G Thomas, G Doussinault, M Trottet \\ INRA, station d'amélioration des plantes, domaine de la Motte, BP 29, 35650 Le Rheu, France
}

(Reçu le 20 mars 1990; accepté le 29 janvier 1991)

\begin{abstract}
Résumé - Pour étudier l'intérêt de la sélection récurrente sur une espèce autogame stricte, le blé tendre, un schéma d'amélioration en population est appliqué à une population à 16 parents. II est basé sur la succession de cycles courts de 3 générations, chacun comprenant 2 générations de sélection et 1 génération d'intercroisement des génotypes sélectionnés. La sélection au cours des générations d'autofécondation est réalisée à l'aide d'index de sélection phénotypique combinant de nombreux caractères pour l'amélioration globale du blé. L'étude des 2 premiers cycles est réalisée en comparant la population finale $\mathrm{PA}_{1}$ à la population initiale $\mathrm{PA}_{0}$ ou à l'ensemble des parents de départ de $\mathrm{PA}_{0}$. Elle montre l'efficacité d'un tel processus en mettant en évidence en particulier une augmentation des valeurs moyennes, après les 2 cycles, pour la plupart des caractères sélectionnés. Par ailleurs, la variabilité intrapopulation, mesurée à partir des variances phénotypiques (et génétiques dans $\mathrm{PA}_{1}$ ), reste sensiblement au niveau initial, sauf pour les caractères fortement sélectionnés pour lesquels elle tend à diminuer. Ce bilan est discuté, notamment pour améliorer le mode d'utilisation d'un schéma de sélection récurrente dans une stratégie générale d'amélioration du blé tendre.
\end{abstract}

sélection récurrente / index de sélection / population de base / variabilité génétique / progrès génétique

Summary - Breeding methodology in soft wheat (Triticum aestivum L). II. Study and analysis of two cycles of a recurrent selection scheme. In order to study recurrent selection effects on soft wheat, a strictly self-fertilizing species, a recurrent breeding scheme was applied to a 16-parent population. It was based on a 3-generation cycle including 2 generations of selection and 1 intercrossing generation of the best genotypes. Selection based on selfing regime was performed with phenotypic indices which combine the main characters introduced in general wheat breeding. The analysis of the 2 first cycles was based on the comparison of the final population $\left(P A_{1}\right)$ with the initial population $\left(P A_{0}\right)$ or with the 16-parent group introduced in $P A_{0}$. It shows the efficiency of such a scheme with an increase in mean level for most characters studied. Moreover, the intrapopulation variability estimated by phenotypic variances (and genetic variances for $P A_{1}$ ) remained at the same level except for the characters that were intensively selected. These evaluations provide a basis for discussion on a better way to insert and apply recurrent selection in a wheat breeding strategy.

recurrent selection / genetic variability / genetic gain / selection index

\section{INTRODUCTION}

Afin d'augmenter la probabilité d'obtenir des génotypes hautement performants et éviter de restreindre la variabilité existant dans les populations, dès le début du XXe siècle s'est développée l'amélioration en populations par sélection récurrente (Hayes et Garber, 1919; East et Jones, 1920). Basé sur l'alternance de phases de sélection (choix des meilleurs génotypes) et de recombinaison (intercroisement des meilleurs génotypes), ce mode d'amélioration a concerné, en premier lieu, les espèces allogames. Procédant par cycles successifs définis par les 3 opérations (obtention, évaluation puis recombinaison des meilleures descendances), la sélection récurrente offre de nombreuses modalités définies selon le système de choix et de recombinaison des unités en sélection (Gallais, 1977; Frey, 1981). 
En se basant sur l'efficacité d'un tel processus (amélioration des performances moyennes et maintien de la variabilité), Gallais (1977) propose une stratégie intégrée de sélection combinant dans un même schéma l'amélioration de populations par récurrence et la création variétale (lignées, hybrides, etc) par des méthodes classiques n'interférant pas directement dans le processus d'amélioration à long terme de la population.

Ce type de stratégie utilisant la sélection récurrente sur des cycles courts (alternance systématique sélection-intercroisements) concerne, en premier lieu, les espèces allogames. Toutefois, le sélectionneur d'espèces autogames est lui aussi confronté à ce double objectif : maintien de variabilité et augmentation de performances. L'autofécondation naturelle après intercroisement initial conduit à l'homozygotie et limite les recombinaisons (Doggett et Eberhart, 1968). De même, la difficulté de réalisation des hybridations conduit à limiter le matériel de départ donc la variabilité exploitée. À l'exemple des schémas allogames, s'est donc développé un courant de recherches visant à adapter à la biologie autogame les méthodes de sélection récurrente à cycle court, basées sur 2 principes :

- partir d'une base génétique la plus large possible dont on limitera la perte de variabilité;

- favoriser régulièrement les recombinaisons pour contrebalancer les effets de l'autofécondation.

Les spécificités des méthodes de la sélection récurrente sur autogames sont dues à la fois à des particularités biologiques (biologie florale) et génétiques (conséquences de l'autofécondation).

\section{MATÉRIEL ET MÉTHODES}

Les principes et méthodes de sélection récurrente appliquée à la population de blé tendre PA, sont les suivants :

\section{La population PA, matériel de base et stratégie générale}

Inspirées des schémas de sélections des espèces allogames, les méthodes de sélection récurrente pour l'amélioration en population nécessitent la prise en compte d'une large variabilité de départ. Chez une espèce autogame comme le blé, la population nommée
PA, outil de base méthodologique, a été créée par hybridations manuelles, en système pyramidal. La population, son origine, son mode de réalisation et ses caractéristiques ont été précisées dans une étude préalable (Thomas et al, 1991). Nous rappellerons simplement ici son origine à 16 parents, choisis pour leurs complémentarités agronomiques et technologiques afin de disposer d'une large variabilité initiale.

Le programme méthodologique entrepris conjointement par les laboratoires INRA de Rennes, ClermontFerrand et GIS Moulon inclut plusieurs volets de la méthodologie d'amélioration du blé. L'objectif agronomique lié à cette étude est l'amélioration de l'adaptation globale des génotypes aux conditions de culture de la moitié Nord de la France.

Dans ce travail, c'est une stratégie complète d'amélioration en population qui a été appliquée sur la population PA à partir des théories de Gallais (1977). Cette stratégie intègre, en particulier, une amélioration de la population PA selon un axe central de sélection récurrente à cycle court. Chaque population PAi (selon le numéro $i$ du cycle) constitue un réservoir de variabilité sans cesse améliorable et peut constituer à tout moment le point de départ d'une sélection "classique" pour créer des lignées fixées, variétés potentielles (fig 1). Dans l'étude, cette sortie variétale en "aval» de la population constitue le deuxième volet méthodologique puisqu'il comprend une comparaison de 4 méthodes de fixation (sélection généalogique, méthode bulk, méthode single seed descent ou SSD, méthode d'haploidiploïdisation) à chaque cycle. Cette analyse comparative fait l'objet d'une étude séparée.

En amont de l'axe d'amélioration de la population, une injection de variabilité nouvelle est possible dans les différentes populations PAi (injection directe, par croisement avec une autre population, introduction de matériel dans une population tampon puis recroisement avec la population PAi). Ceci constitue un volant de manœuvre permettant de rectifier certains défauts au fur et à mesure de l'évolution sous l'effet de l'amélioration en population.

La présente étude s'inscrit dans cette stratégie générale d'amélioration de la population PA mais concerne seulement l'axe central de cette stratégie, c'est-à-dire la mise en œuvre et l'analyse d'un processus de sélection récurrente pour le blé, espèce autogame.

\section{Description du schéma de sélection récurrente appliqué à la population PA}

Basé sur l'alternance de phases de sélection et d'intercroisement, chaque cycle de sélection récurrente comprend 3 générations:

- deux générations de sélection avec reproduction par autofécondations;

- une génération d'intercroisement des génotypes sélectionnés précédemment. 


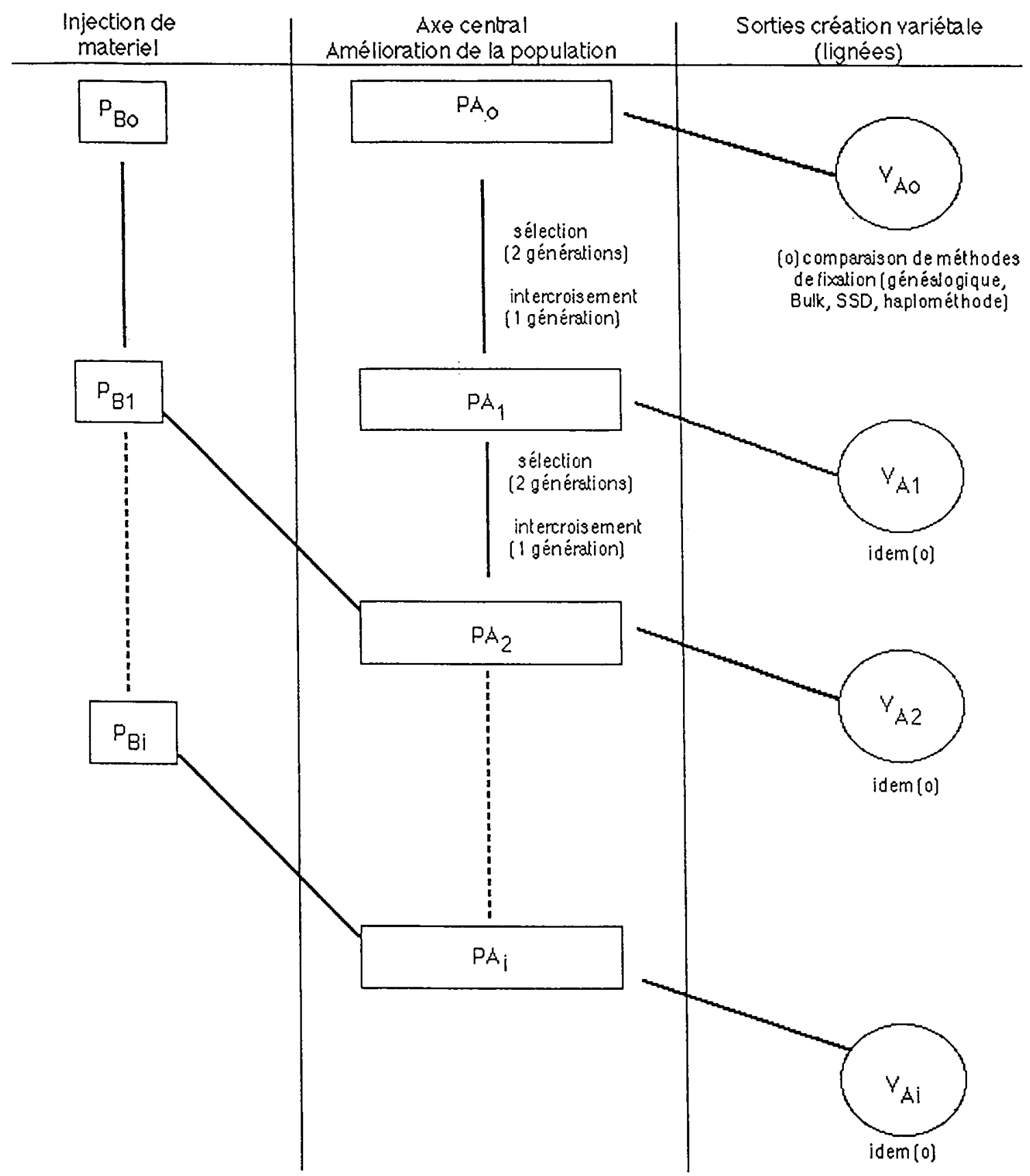

Fig 1. Stratégie générale d'amélioration de la population PA (adaptée d'après Gallais, 1977).

Les 2 types d'opérations seront décrits successivement.

\section{Sélection}

\section{Type et taux de sélection}

Deux générations successives permettent le choix d'individus parentaux pour les recroisements de fin de cycle. Cette sélection se fait sur 2 générations d'autofécondations $S_{0}$ et $S_{1}$, distinctes selon le type d'unités sélectionnées:

- en $S_{0}$, sélection phénotypique individuelle unilocale
- en $S_{1}$, sélection plurilocale combinée individufamille (descendance de plante) : choix des meilleurs individus dans les meilleures familles pour l'intercroisement.

Compte tenu de la diversité de leurs natures, nous retiendrons le terme générique d'unités en sélection pour désigner les individus et (ou) les familles sélectionnées.

Du fait de l'objectif de sélection récurrente, le taux de sélection appliqué est volontairement faible. À titre indicatif, le tableau I fait apparaître les variations du taux de sélection pour $\mathrm{PA}_{0}$ et $\mathrm{PA}_{1}$ et montre un taux individuel modéré (nécessité de faire un progrès) et 
Tableau I. Taux de sélection appliqués à $\mathrm{PA}_{0}$ et $\mathrm{PA}_{1}$ pour les 2 premiers cycles.

\begin{tabular}{rcccccc}
$\begin{array}{c}\text { Nature } \\
\text { matériel }\end{array}$ & $\begin{array}{c}\text { Effectif } \\
\text { initial } \\
\text { individus }\end{array}$ & $\begin{array}{c}\text { Effectif } \\
\text { sélection } \\
\text { individus }\end{array}$ & $\begin{array}{c}\text { Effectif } \\
\text { initial } \\
\text { familles }\end{array}$ & $\begin{array}{c}\text { Effectif } \\
\text { sélection } \\
\text { familles }\end{array}$ & $\begin{array}{c}\text { Taux } \\
\text { individus } \\
(\%)\end{array}$ & $\begin{array}{c}\text { Taux } \\
\text { familles } \\
(\%)\end{array}$ \\
\hline $\mathrm{PA}_{0} \mathrm{~S}_{0}$ & 1551 & 464 & 240 & 163 & 29,92 & 67,90 \\
$\mathrm{~S}_{1}$ & $464^{*}$ & $80^{* *}$ & $163^{*}$ & $61^{* *}$ & 17,24 & 76,25 \\
$\mathrm{PA}_{1}$ & 1798 & 149 & 221 & 149 & 8,30 & 34,80 \\
$\mathrm{~S}_{0}$ & $148^{*}$ & $80^{* *}$ & $149^{*}$ & $80^{* *}$ & 5,40 & 53,70 \\
$\mathrm{~S}_{1}$ & & & & & & \\
\hline
\end{tabular}

"Effectif présent par lieu.* Effectif retenu par lieu.

un taux familial faible (nécessité de maintien de la variabilité).

\section{Caractères et dispositifs de sélection}

Le nombre de caractères pris en compte est important du fait de l'objectif d'amélioration fixé (adaptation globale aux conditions de culture de la moitié Nord de la France). Ceci a conduit à l'analyse, dans divers dispositifs, de caractères très variés :

- en pépinière semée grain à grain, étude de l'ensemble des caractères de la première génération $S_{0}$ permettant l'analyse sur des plantes isolées, semées par origine sur des lignes de $1,50 \mathrm{~m}$ (11 grains par ligne; écartement entre lignes de 0,25 m);

- en essai à densité forte, appréciation du matériel en familles de plantes sur les caractères de rendement et de qualité à héritabilité faible. Ces essais (à la génération $\mathrm{S}_{1}$ ) ont été menés selon un dispositif spécifique faisant intervenir chaque famille à raison de 2 répétitions constituées d'une ligne chacune, à densité normale de culture (50 grains sur $1,50 \mathrm{~m}$ ).

Dans ces 2 types d'essais, les caractères analysés peuvent être répartis en 3 groupes :

\section{Caractères d'adaptation biologique}

- appréciation de résistance aux maladies : oïdium du feuillage (code $\left.O I D_{1}\right)$ et des épis $\left(O I D_{2}\right)$, rouilles jaune $(R J)$, brune $(R B)$ et noire $(R N)$, septoriose (S), piétin verse $(P V)$;

- appréciation de la hauteur des plantes (en liaison avec la verse) (HA) et du rapport distance dernière feuille-épi/hauteur totale (Rc).

\section{Caractères de productivité}

- mesure du rendement (lignes à densité normale de culture) (RDT);
- mesure des composantes du rendement (nombre d'épillets (NET), nombre de grains contenus dans 3 épis d'une plante $\left(N G_{3} E\right)$, poids de grain dans 3 épis $\left(P G_{3} E\right)$, poids de 1000 grains (PMG).

\section{Caractères de qualité}

- mesure par des tests indirects (test Zeleny (ZEL); Pelshenke (PEL));

- mesure de la teneur en protéines $(N 5,7)$.

L'ensemble des caractères analysés, leur mode de mesure et le dispositif de mesure figurent au tableau II.

\section{Mode de sélection multicaractères}

Les divers caractères précités doivent être combinés pour une même génération de sélection $\left(\mathrm{S}_{0}\right.$ (et) ou $\left.\mathrm{S}_{1}\right)$ de chacun des cycles. Parmi les méthodes de sélection multicaractères possibles (tandem, parallèle, niveaux indépendants, index), le principe de la sélection sur index a été retenu afin, notamment, d'étudier l'efficacité de cet outil de sélection pour l'espèce considérée.

Les index employés sont des index phénotypiques, chaque individu constituant une combinaison linéaire de valeurs phénotypiques des unités (individus ou familles) en sélection. Les unités sont sélectionnées sur les plus faibles valeurs d'index.

\section{Mode d'appréciation de la valeur phénotypique $\mathrm{Xa}$}

Pour chaque caractère, la valeur brute mesurée $X_{a i}$ peut être transformée avec une simple pondération $X_{a i}$ $=K_{a} \cdot X_{\hat{a}}$. Elle peut aussi être rapportée à une valeur optimale ou maximale $X_{a 0}$ fixée selon l'objectif de sélection pour le caractère étudié. Dans ce cas, $X_{a i}=$ 
Tableau II. Description et mesure des caractères étudiés dans la population PA.

\begin{tabular}{|c|c|c|c|c|c|}
\hline \multirow{3}{*}{$\begin{array}{l}\text { Caractère } \\
\text { Oïdium feuilles } \\
\text { Oïdium épis }\end{array}$} & \multirow{3}{*}{ Abréviation } & \multirow{3}{*}{$\begin{array}{l}n \\
\text { Méthode de mesure } \\
\text { Échelle } 1-9(\% \text { feuillage contaminé }) / p l t e ~ o u ~ f a m i l l e) \\
\text { Échelle } 1-9 \%(\% \text { des épis contaminés/plte ou par famille) }\end{array}$} & \multicolumn{3}{|c|}{ Dispositif utilisé } \\
\hline & & & \multicolumn{3}{|c|}{ Pépinière grain à grain } \\
\hline & & & $"$ & $"$ & $"$ \\
\hline Rouille jaune & RJ & $\begin{array}{l}\text { Échelle } 1-9 \text { (intensité attaque en \% feuillage contaminé) } \\
\text { juxtaposée à échelle } 1-4 \text { (/ type d'infection) }\end{array}$ & $"$ & " & $"$ \\
\hline Rouille brune & $R B$ & $\begin{array}{l}\text { Échelle } 1-9 \text { (intensité attaque en \% feuillage contaminé) } \\
\text { juxtaposée à échelle } 1-4 \text { (type d'infection par plante } \\
\text { ou par famille) }\end{array}$ & $"$ & $"$ & $"$ \\
\hline Rouille noire & $R N$ & $\begin{array}{l}\text { Échelle } 1-9 \text { (intensité attaque en \% feuillage contaminé) } \\
\text { juxtaposée à échelle } 1-4 \text { (type d'infection par plante } \\
\text { ou par famille) }\end{array}$ & $"$ & $"$ & $"$ \\
\hline Septoriose & $S$ & Échelle $1-9$ sur \% contamination d'épis/plte : & $"$ & $"$ & $"$ \\
\hline Hauteur & $H A$ & Longueur de la base de l'épi à la base de la plante $(\mathrm{e} \mathrm{cm})$ & $"$ & $"$ & $"$ \\
\hline Rapport $R c$ & & Rapport longueur dernière feuille. épi/hauteur totale HA & $"$ & $"$ & $"$ \\
\hline Précocité & $E P$ & $\begin{array}{l}\text { Précocité d'épiaison mesurée en écarts de nombre } \\
\text { des jours par rapport au premier génotype épié dans la populati }\end{array}$ & tion & $n$ & $"$ \\
\hline Nombre d'épillets & $N E T$ & $\begin{array}{l}\text { Moyenne du nombre d'épillets à partir de } \\
3 \text { épis par plante }\end{array}$ & " & " & $"$ \\
\hline $\begin{array}{l}\text { Poids de } \\
3 \text { épis entiers }\end{array}$ & $P_{3} E E$ & Valeur du poids de 3 épis entiers non battus par plante & " & $"$ & $"$ \\
\hline $\begin{array}{l}\text { Poids de grain } \\
\text { de } 3 \text { épis }\end{array}$ & $P G_{3} E$ & Valeur du poids de grain de 3 épis battus par plante & $"$ & $"$ & $"$ \\
\hline $\begin{array}{l}\text { Nombre de grains } \\
\text { de } 3 \text { épis }\end{array}$ & $N G_{3} E$ & $\begin{array}{l}\text { Nombre de grains contenus dans } 3 \text { épis } \\
\text { battus par plante }\end{array}$ & $"$ & $"$ & $"$ \\
\hline Poids de 1000 grains & $P M G$ & Poids moyen de 2 lots de 500 grains & Ess & dde & \\
\hline Rendement en ligne & $R D T$ & $\begin{array}{l}\text { Poids moyen de grain récolté sur } 1 \mathrm{~m} \\
{[50 \mathrm{gr} / 1,50 \mathrm{~m}]}\end{array}$ & $\begin{array}{l}\text { Ess } \\
3 \text { li }\end{array}$ & & \\
\hline
\end{tabular}

$K_{a}^{\prime}\left(X_{\hat{a} r} X_{\hat{a} o}\right)$. Ce mode de calcul concerne les caractères de hauteur et de précocité $(H A, E P)$ ainsi que les caractères pour lesquels les valeurs brutes les plus élevées sont les meilleures (caractères de rendement $\left(P_{3} E, N G_{3} E, N E T, P M G, R D T\right)$. On écrit alors $X_{a i}=K_{a}$ $\left(M_{a x} X_{\hat{a}}-X_{\hat{a} i}\right)$.

\section{Choix des pondérations}

Chaque pondération $\mathrm{K}_{\mathrm{a}}$ affectée au caractère a pour double but d'équilibrer la valeur absolue des caractères (en centrant et réduisant les variables de départ $X_{\hat{a} i}$ ) et d'affecter une importance relative différente à chacun des caractères étudiés. Ce deuxième objectif est réalisé par le choix à priori d'une hiérarchie parmi les caractères étudiés lors d'une même génération. Cette hiérarchie est fixée en fonction d'expériences antérieures de la sélection dans l'espèce.

Ces index, utilisés ici en tant qu'outils de sélection récurrente, feront l'objet d'une étude séparée. II faut noter que l'usage d'index partiels permet une sélection par paliers successifs : choix sur des caractères observés au champ donnant lieu à un premier index et à une première sélection puis observation de caractères à la récolte et au laboratoire permettant une deuxième sélection. Cet usage permet aussi le regroupement entre lieux (index partiel par lieu).

Pour chaque génération de sélection, les caractères introduits dans l'index et les modalités de regroupement par index partiels sont récapitulés au tableau III. II fait apparaître une variation selon les générations et les cycles successifs avec un faible nombre de caractères analysés en un seul lieu pour les générations $S_{0}$ et un ensemble de caractères plus important en $S_{1}$, hiérarchisés par index partiels et par lieu (tableau III et fig 2).

\section{Intercroisements}

À l'aide des résultats des 2 générations précédemment décrits, sont sélectionnés des génotypes qui 
Tableau III. Modalités de réalisation des index partiels dans la population PA $\mathrm{PA}_{1}$.

\begin{tabular}{|c|c|c|c|}
\hline $\begin{array}{l}\text { Cycle } \\
\text { génération }\end{array}$ & $\begin{array}{l}\text { Index partiel } 1 \\
\text { caractères }\end{array}$ & Index partiel 2 & Index global \\
\hline $\mathrm{S}_{0}$ & $\begin{array}{l}\text { au champ }=\text { INDAGRON } \\
O D_{1}, R J, R B, R N, H A, R_{C}\end{array}$ & $\begin{array}{l}\text { après récolte }= \\
\text { INDREC } \\
(\text { FERT, ÉCH, VITR, } \\
\text { COUL + INDRAGON) }\end{array}$ & $\begin{array}{l}\text { INDREC } \\
1 \text { lieu }\end{array}$ \\
\hline $\mathrm{S}_{1}$ & $\begin{array}{l}\text { INDAGRON } \mathrm{S}_{1} \\
\text { INDAGRON S}_{0}, \mathrm{PV}, \\
\mathrm{OID}_{1}, \mathrm{~S}, \mathrm{HA}, \mathrm{ECH}, \mathrm{Rc}, \mathrm{RDT}\end{array}$ & $\begin{array}{l}\text { INDTECNO } \\
P E L S, Z E L, N 5,7\end{array}$ & $\begin{array}{l}\text { INDAGRON } \\
+ \\
\text { INDTECNO } \\
3 \text { lieux }\end{array}$ \\
\hline$S_{0}$ & $\begin{array}{l}\text { INDAGRON } \\
\text { OID, RJ, RB, HA }\end{array}$ & $P_{3} E$ & $\begin{array}{c}\text { INDAGRON } \\
+ \\
P_{3} E \\
1 \text { lieu }\end{array}$ \\
\hline$S_{1}$ & $\begin{array}{l}\text { INDAGRON } \\
E P, O I D_{1}, O I D_{2}, R B, H A, R c \\
P_{3} E\end{array}$ & $\begin{array}{l}\text { INDPROD } \\
P_{3} E, R D T\end{array}$ & $\begin{array}{ll}\text { INDTECNO INDTECNO } \\
\text { PELS, ZEL, INDAGRON } \\
\text { N5,7 } & \text { INDPROD } \\
& 3 \text { lieux }\end{array}$ \\
\hline
\end{tabular}

vont participer à l'intercroisement terminal de chaque cycle. Comme pour la création de la population de départ, ces croisements sont réalisés par castration manuelle et sont limités à une seule génération. Les candidats à l'intercroisement sont représentés par des familles sélectionnées sur index, communes à chaque lieu : chaque famille y est prise en compte une fois comme femelle et une fois comme mâle. Chaque famille retenue fournit 3 individus servant de femelles et 3 individus servant de mâles et participe ainsi à 6 croisements différents. Dans la réalisation des intercroisements, 2 étapes seront présentées :

\section{Réalisation du plan de croisement}

Ce plan gérant les modalités de croisement des unités retenues dans la population PAi pour la réalisation de la population $\mathrm{PA}(i+1)$ est réalisé en 3 parties correspondant aux unités retenues dans chacun des 3 lieux de sélection. Deux règles principales servent à l'élaboration des plans:

- réaliser les appariements en évitant d'associer 2 familles ayant les mêmes défauts. Ces défauts sont évalués sur différents caractères en sélection et sont attribués à un génotype donné en dessous d'un seuil minimal;

- réaliser les appariements en évitant de croiser 2 familles apparentées. Les familles sont reconnues comme telles si elles proviennent toutes 2 du même individu de la population $\mathrm{PA}_{i} \mathrm{~S}_{0}$.

Les appariements, hormis ces règles, sont réalisés au hasard; chaque lieu gère son propre plan et la population $\mathrm{PA}(i+1)$ résulte de la cumulation des 3 plans locaux.

\section{Réalisation de la population}

La population $\mathrm{PA}(i+1)$ est réalisée par castration manuelle de 2 épis femelles de chaque famille sélectionnée. Ces épis sont pollinisés par 3 épis mâles issus de la même famille mâle. Quatre-vingt croisements par lieu permettent à chaque cycle de produire une nouvelle population $\mathrm{PA}(i+1)$ avec 240 génotypes théoriques de base.

\section{Modes d'évaluation de la sélection récurrente}

Pour les 2 cycles analysés, l'étude porte sur les résultats et effets de la sélection récurrente. Afin de prendre en compte les fluctuations interannuelles d'estimation des caractères (variations des infestations parasitaires, du climat), l'évolution de la population PA a été étudiée en comparant les performances, pour une même année, des populations successives. Les différentes populations sont examinées selon les 2 types de dispositifs décrits dans ces 8 caractères et dispositifs de sélection, dans la même pépinière et la même année. Ainsi, ont été conduits parallèlement aux populations $S_{0} P A_{1}$ et $S_{1} P A_{1}$, des échantillons représentatifs de la population $\mathrm{PA}_{0}$ (100 individus). Ceci a permis de comparer dans les mêmes conditions, les 2 générations successives $S_{1}$ et $S_{1}$ des 2 cycles $P A_{0}$ et $P A_{1}$. Dans le même but, ont été comparées les performances des populations $\mathrm{PA}_{0}$ et $\mathrm{PA}_{1}$ avec l'ensemble des 16 parents de PA (moyenne nommée $P_{0}$ ). Les 2 populations de référence permettent pour chaque ca- 


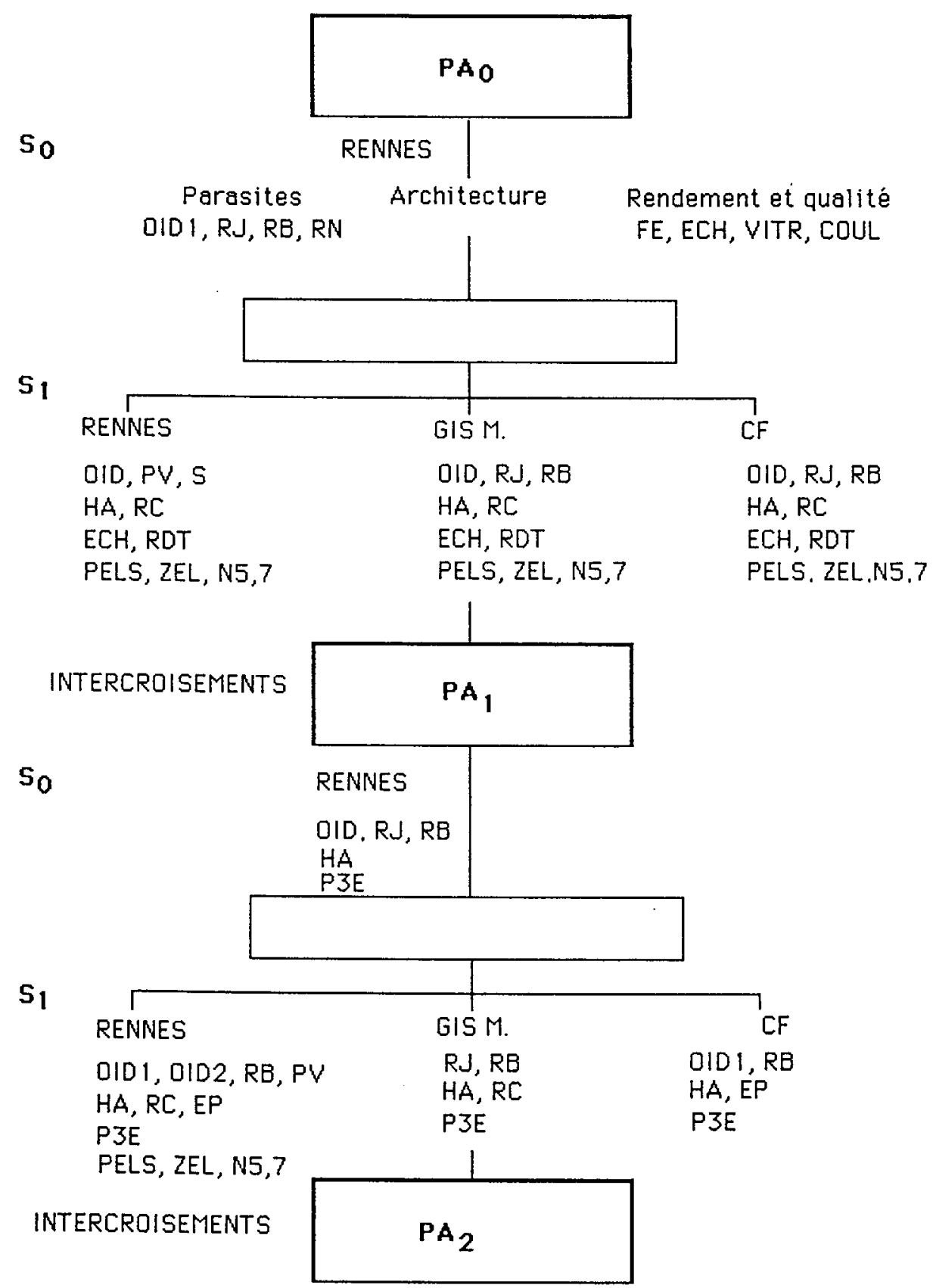

Fig 2. Population PA. Stratégie générale de sélection récurrente et choix des caractères en sélection.

ractère de calculer un terme $P$ de progrès résultant $d u$ rapport ayant pour valeur absolue :

$|P|=\mid$ moyenne population $\mathrm{PA}_{1}-$ moyenne population référence| / |moyenne population référence|

On attribue à ce rapport $P$ un signe :

- positif si la performance de $\mathrm{PA}_{1}$ est supérieure à la performance de $\mathrm{PA}_{0}$ (dans le sens du progrès même si le caractère est de type inversé ie valeurs fortes favorables);

- négatif si la performance de $\mathrm{PA}_{1}$ est inférieure à la performance de $\mathrm{PA}_{0}$.

L'ensemble de cette analyse comparative, conduite à Rennes, s'est basée sur les caractères inclus dans la sélection sur index à partir de principes classiques d'analyse de variance. Pour les générations $P_{0}$ et $P A_{1} S_{1}$, les variances génétiques familiales ont été estimées à l'aide de la bibliothèque statistique Amance (Bachacou et al, 1981).

\section{RÉSULTATS \\ ANALYSE DE LA POPULATION PA EN SÉLECTION RÉCURRENTE}

L'analyse est basée sur la comparaison des populations de référence $\mathrm{PA}_{0}$ ou $\mathrm{P}_{0}$ avec la popu- 
lation $\mathrm{PA}_{1}$ dans les conditions de Rennes. Nous étudierons successivement l'évolution quantitative des caractères entre les 2 cycles en examinant de façon dissociée les 2 générations (comparaison $S_{0} P A_{0}-S_{0} P A_{1}$ et $S_{1} P A_{0}-S_{1} P A_{1}$ ).

\section{Évolution qualitative des caractères}

\section{Moyennes}

Le tableau IV présente l'évolution des moyennes et des variances pour les différents caractères intégrés dans les index de sélection. Une modification de la moyenne dans le sens du progrès est observée pour la plupart des caractères, dans la génération $S_{0}$, confirmée dans la génération $\mathrm{S}_{1}$. Cette modification se traduit par une différence de moyenne modérée pour les caractères tels que maladies $\left(O I D_{1}, P V\right)$, rendement $\left(N G_{3} E, P M G, N E T\right)$ et plus importante pour d'autres critères (maladies : $R B, R N, R J)$, hauteur (HA, Rc).

\section{Histogrammes fréquentiels}

L'étude des moyennes peut être complétée par l'analyse comparée de la distribution en classes de fréquence pour chacun des caractères. Pour les 2 générations et les 2 cycles, 3 types de dis-

Tableau IV. Performances comparées des populations $\mathrm{PA}_{0}$ et $\mathrm{PA}_{1}$ pour les 2 générations de sélection.

Génération $P A-S_{0}$ (année 1981-1982)

\begin{tabular}{|c|c|c|c|c|c|c|}
\hline & & \multicolumn{4}{|c|}{ Caractères étudiés } & \multirow[b]{2}{*}{$\begin{array}{l}\text { Poids } 3 \text { épis } \\
\quad P G_{3} E \\
\text { eng }\end{array}$} \\
\hline & & $\begin{array}{c}\text { Ödium feuilles } \\
\text { OID } \\
\text { Ech } 1-9\end{array}$ & $\begin{array}{l}\text { Rouille jaune } \\
\text { RJ } \\
\text { EC } 1-9\end{array}$ & $\begin{array}{c}\text { Rouille brune } \\
\text { RB } \\
\text { Ech } 1-9\end{array}$ & $\begin{array}{l}\text { Hauteur } \\
\mathrm{HA} \\
\text { en } \mathrm{cm}\end{array}$ & \\
\hline \multirow{2}{*}{$\begin{array}{l}\text { Moyenne } \\
\text { population } \\
\qquad \bar{x}\end{array}$} & $\mathrm{PA}_{1}$ & 3,52 & 1,68 & 1,90 & 86,64 & 5,24 \\
\hline & $\mathrm{PA}_{0}$ & 3,44 & 2,62 & 2,98 & 96,16 & 4,70 \\
\hline \multirow{2}{*}{$\begin{array}{c}\text { Variance } \\
\text { population } \\
\sigma^{2}\end{array}$} & $\mathrm{PA}_{1}$ & 1,02 & 1,29 & 2,28 & 165,88 & 4,56 \\
\hline & $\mathrm{PA}_{0}$ & 0,50 & 1,66 & 3,12 & 126,56 & 4,30 \\
\hline \multirow{2}{*}{$\begin{array}{c}\text { Écart type } \\
\text { population } \\
\sigma\end{array}$} & $\mathrm{PA}_{1}$ & 1,01 & 1,14 & 1,51 & 12,28 & 2,14 \\
\hline & $\mathrm{PA}_{0}$ & 0,70 & 1,29 & 1,77 & 11,25 & 2,07 \\
\hline
\end{tabular}

Générations $P A-S_{1}$ (année 1982-1983)

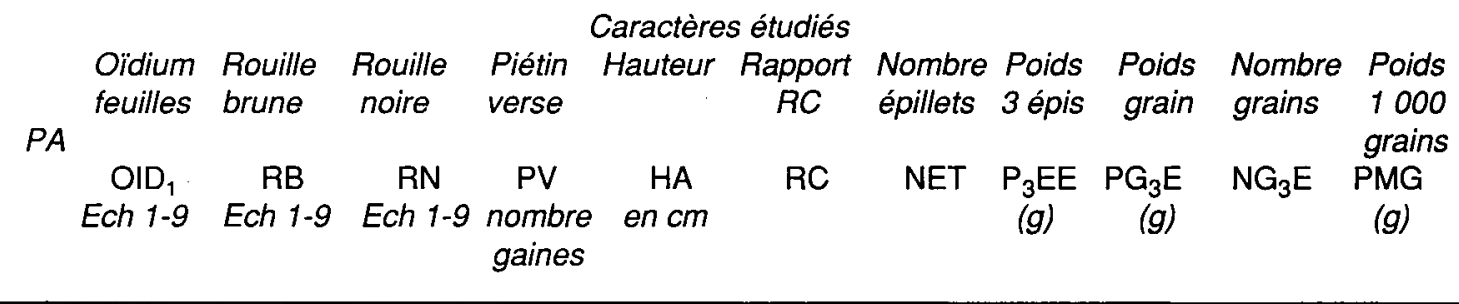

\begin{tabular}{clllrlrllllll}
\hline & & & & & & & & & & & \\
Moyennes & $\mathrm{PA}_{1}$ & 2,90 & 4,81 & 1,12 & 2,10 & 71,10 & 0,110 & 20,79 & 6,66 & 4,18 & 135,92 & 29,64 \\
$\bar{x}$ & $\mathrm{PA}_{0}$ & 3,46 & 7,24 & 3,64 & 2,30 & 73,34 & 0,143 & 19,17 & 7,02 & 4,31 & 133,00 & 29,20 \\
& & & & & & & & & & & & \\
Variance & $\mathrm{PA}_{1}$ & 1,03 & 26,45 & 0,17 & 0,70 & 72,62 & 0,035 & 1,70 & 1,61 & 1,35 & 993,1 & 13,91 \\
$\sigma^{2}$ & $\mathrm{PA}_{0}$ & 0,91 & 52,36 & 40,00 & 1,40 & 144,20 & 0,002 & 3,67 & 3,36 & 2,87 & 1334,9 & 3,53 \\
& & & & & & & & & & & & \\
Écart type & $\mathrm{PA}_{1}$ & 1,00 & 5,14 & 0,41 & 0,85 & 8,52 & 0,190 & 1,31 & 1,27 & 1,16 & 31,51 & 3,73 \\
$\sigma$ & $\mathrm{PA}_{0}$ & 0,97 & 7,34 & 6,41 & 1,10 & 12,18 & 0,046 & 1,94 & 1,86 & 1,72 & 37,10 & 1,88 \\
\hline
\end{tabular}



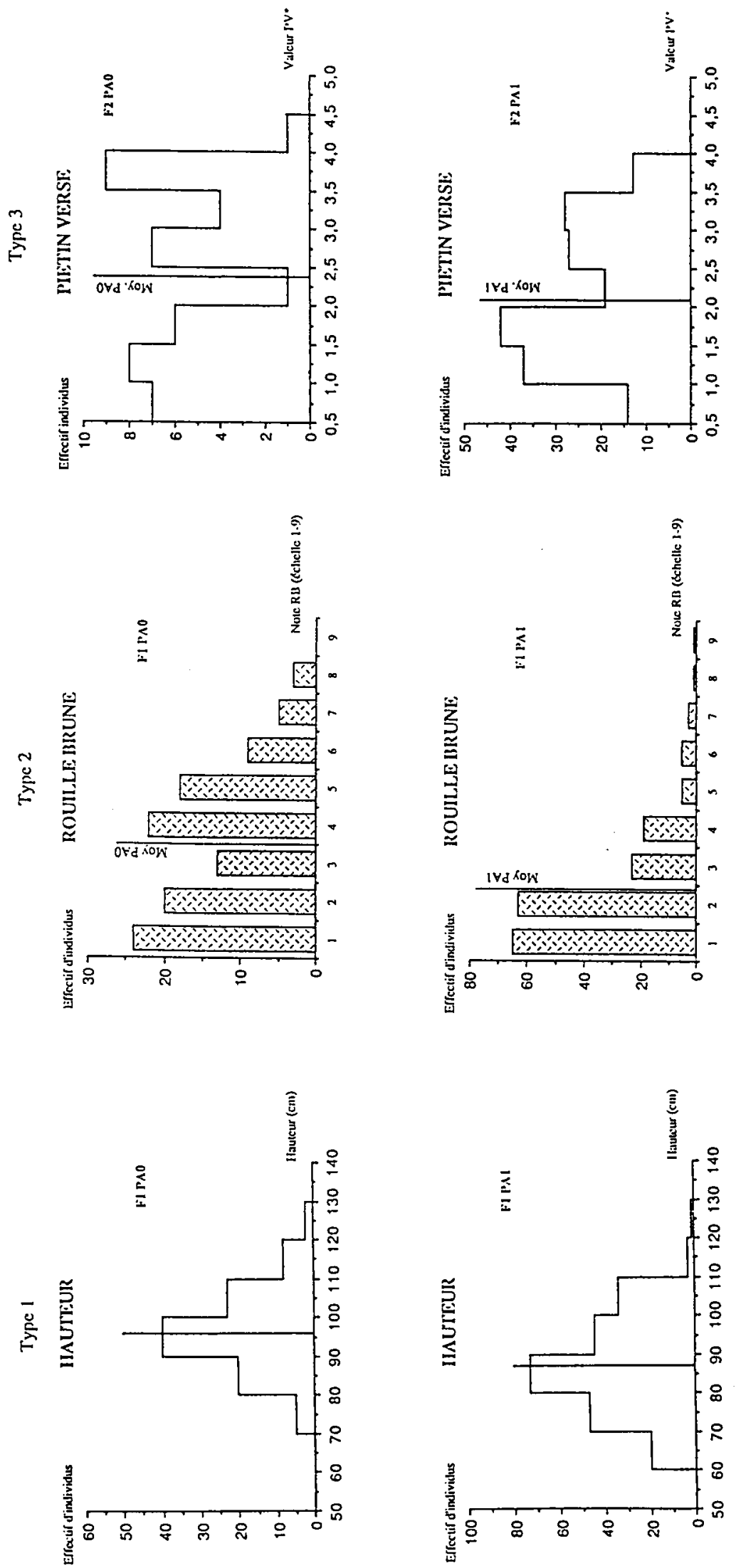
tribution peuvent être rencontrés, illustrés chacun par un exemple graphique (fig 3 ).

\section{Type 1}

Distribution de type normal $\left(O I D_{1}, P G_{3} E, H A\right.$, $\left.N E T, N G_{3} E, P M G\right)$. Les valeurs mesurées, pour ces caractères, se répartissent autour de la valeur moyenne de la population avec un simple décalage global de l'ensemble de l'histogramme entre $P A_{0}$ et $P A_{1}$.

\section{Type 2}

Distribution unimodale (valeurs négatives inexistantes) pour $R J, R B$. Du fait d'une importante réduction de moyenne entre $\mathrm{PA}_{0}$ et $\mathrm{PA}_{1}$, la distribution de $P A_{1}$ est entièrement décalée vers l'origine avec un effectif important de génotypes proches de la valeur nulle (individus résistants ou très peu sensibles).

\section{Type 3}

Distribution bimodale (caractère PV) avec 2 pics de fréquence élevée (l'un vers les valeurs très faibles, l'autre vers les valeurs fortes correspondant aux types sensibles).

Ces 3 types de distribution correspondent à des caractères introduits de déterminisme génétique différent. Pour le type 1 , ils sont à hérédité complexe quantitative. L'amélioration en un cycle ne peut être que limitée et explique la faible modification des histogrammes. Les caractères relevant du type 2 permettent d'obtenir facilement des génotypes à valeur extrême réunissant tous les allèles favorables. Lorsqu'on sélectionne sur quelques gènes majeurs, la distribution se trouve entièrement décalée en retenant les génotypes résistants. Pour le type 3, la présence d'un gène majeur de résistance correspondant au groupe incluant la résistance du géniteur VPM (parents $\mathrm{VP}_{4}, \mathrm{VM}_{4}, \mathrm{~V}_{2} \mathrm{D}_{11}$ ) correspond à la première partie de la courbe bimodale, la deuxième étant représentée par l'ensemble des autres génotypes de la population n'incluant aucun facteur de résistance.

\section{Appréciation quantitative de la sélection récurrente}

II importe de pouvoir quantifier le progrès réalisé et comparer les différents caractères par rapport à l'analyse ci-dessus. Cette étude comparative sera faite par l'étude du progrès $P$ calculé avec les 2 références $P A_{0}$ et $P_{0}$ (voir mode d'évaluation de la sélection récurrente).

\section{Progrès sur le niveau de la population}

\section{Population de référence $P A_{O}$}

Pour la génération $S_{0}$ (tableau $V$ ), un rapport $P$ élevé, signe d'un progrès important, est observé pour la résistance aux rouilles. Pour les autres caractères, des progrès plus faibles (environ $10 \%$ ) sont enregistrés $\left(H A, P G_{3} E\right)$ alors qu'une régression est même notable pour la résistance à l'oïdium $\left(O I D_{1}\right)$. Pour la génération $S_{1}$, on opposera 2 types de caractères : ceux, en majorité, qui présentent une certaine amélioration (dans l'ordre de progrès décroissant $R N, P M G, R B, S$, $\left.O I D_{1}, P V, N E T, H A, N G_{3} E\right)$ à ceux donc les performances ont régressé $\left(P_{3} E E, P G_{3} E, R c\right)$.

\section{Population de référence $P_{0}$}

L'usage de cette référence modifie l'évaluation des performances moyennes de PA. Nous ne noterons que les différences de comportement correspondant à l'examen de ce deuxième mode d'évaluation.

Pour la génération $S_{0}$, on observe une régression pour la hauteur $(H A)$ révélant une absence de progrès pour cette seule génération et ce caractère, sans doute due à une sous-estimation de la taille des lignées parentales lors de cette année d'étude (conditions expérimentales défavorables plus marquées sur des lignées). Pour la génération $S_{1}$, la référence $P_{0}$ permet d'étudier le caractère EP pour lequel il $y$ a eu progrès entre les 2 cycles.

Par ailleurs, cette référence modifie les estimations réalisées sur la base de la population $\mathrm{PA}_{0}$ avec, notamment, régression pour la résistance à la septoriose (S) mais par contre, progrès pour des caractères liés au rendement comme $P_{3} E E$ et $P G_{3} E$.

\section{Discussion}

Progrès obtenu après le cycle $P A_{1}$

La sélection récurrente ( 2 générations de sélection et 1 génération d'intercroisement) a été efficace pour de nombreux caractères :

- haute efficacité pour les rouilles, permise par la possibilité de sélectionner des individus immuns ou d'un niveau de résistance élevé et de conserver seulement ces individus, du fait de la forte héritabilité du caractère;

- efficacité modérée pour de nombreux caractères, liée à l'absence de gènes majeurs aisément sélectionnables. Dans ce cas, la sélection 


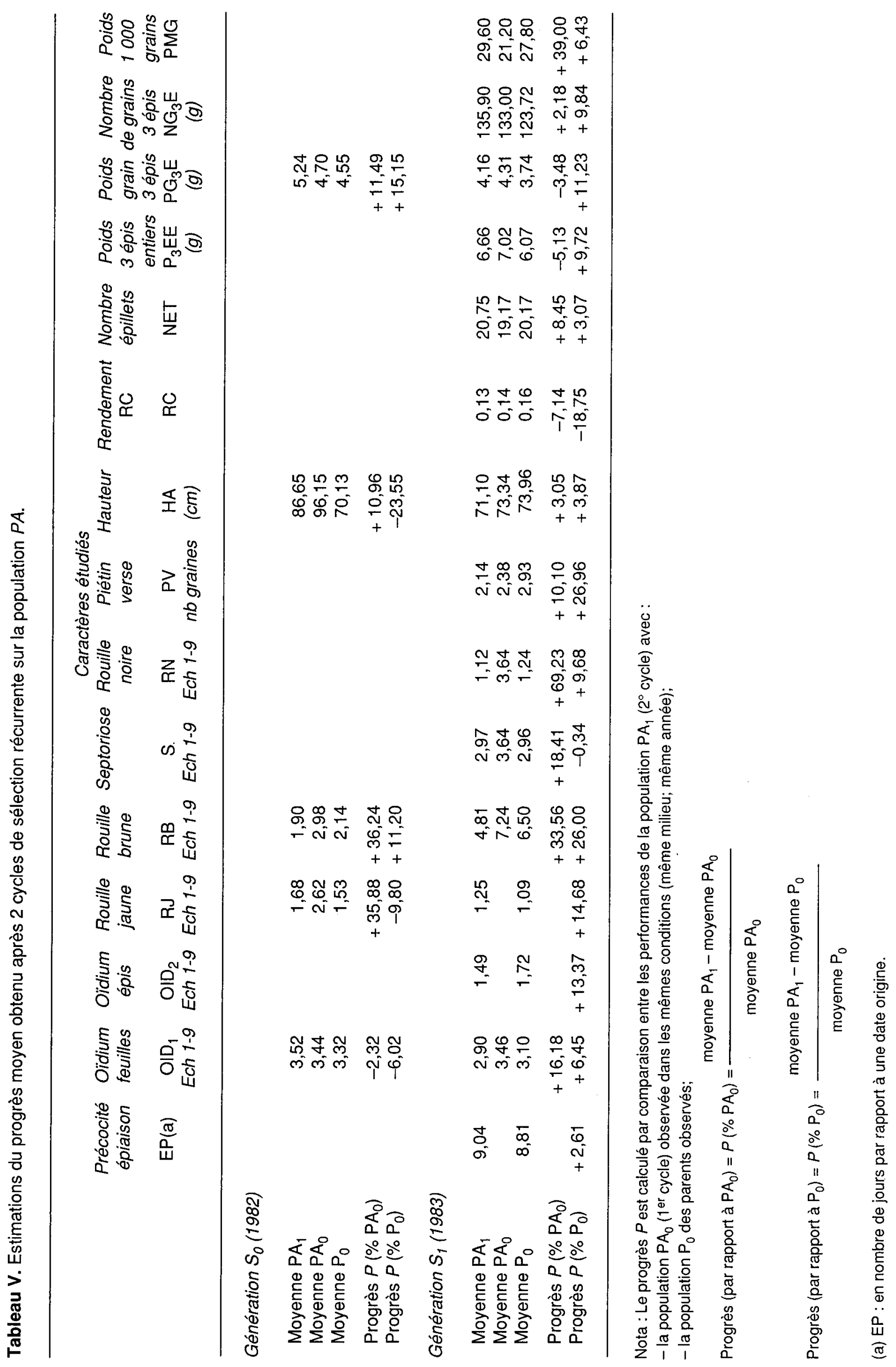


récurrente prend toute son importance permettant, dans les cycles ultérieurs, d'accumuler au fur et à mesure le progrès en sélection.

Pour certains caractères ne manifestant pas de progrès, on peut expliquer ce défaut par un nombre insuffisant de générations et de cycles de sélection ou un changement dans les gènes de virulence présents dans la population de l'agent pathogène (cas de $O I D_{1}, R c$ ) voire même une absence de sélection directe sur ces caractères au cours de ces cycles : sélection sur le rendement dans le premier cycle privilégiant certains de ces paramètres du rendement (PMG, $N G_{3} E, N E T$ mais pas d'autres : $\left.P_{3} E E, P G_{3} E\right)$.

Globalement, pour les caractères étudiés lors des 2 générations de chaque cycle; nous noterons que le progrès évalué à la génération $S_{0}$ est généralement moins important que pour la deuxième génération. Ceci peut être dû à un effet de dilution lié au fait qu'à cette génération, le matériel évalué est très hétérozygote, dérivant immédiatement de l'intercroisement récurrent.

\section{Comparaison des 2 méthodes d'évaluation}

Les estimations du progrès obtenu à partir de chacune des 2 populations de référence diffèrent pour certains caractères et sont essentieliement dues à la nature même des populations de référence : l'une $\mathrm{PA}_{0}$, population au sens strict, très hétérozygote et hétérogène résultant de l'intercroisement pyramidal, l'autre $P_{0}$ résultant de l'assemblage des valeurs des 16 lignées parentales.

Globalement, les progrès enregistrés avec $P_{0}$ sont nettement supérieurs à ceux provenant de $\mathrm{PA}_{0}$, signe d'infériorité de la somme des parents par rapport à $\mathrm{PA}_{0}$ dont les performances résultent de la combinaison de ces 16 parents. Ceci confirme la nécessité de préciser la référence de mesure du progrès en sélection récurrente. Dans ce but, la référence "parents" est disponible à tout moment du schéma de sélection récurrente avec un risque possible de variations interannuelles alors que la référence $P A_{0}$ (ou $P A(i-1)$ nécessite une conservation et expérimentation systématiques des populations antérieures alourdissant le processus. II serait de même possible d'analyser les progrès en sélection récurrente en comparant aux lignées parentales, les meilleures lignées fixées (par haplodiploïdisation par exemple) issues d'un cycle.

\section{Évolution des variances de la population Évaluation des variances}

\section{Variances phénotypiques globales}

L'estimation de la variance phénotypique intrapopulation, évaluée à partir de l'ensemble des individus de chaque population, peut donner d'emblée un aperçu de l'évolution au cours des cycles en comparant $\mathrm{PA}_{0}$ et $\mathrm{PA}_{1}$ dans les mêmes conditions et la même année (tableau IV).

Les variances, en $S_{0}$ et en $S_{1}$, diminuent pour l'ensemble des caractères sauf pour $O I D$ et $R C$ qui restent constants. On peut relier la diminution forte de variance aux caractères qui ont été sélectionnés efficacement $(R B, R J, R N, H A)$ (élévation importante de la moyenne) alors que les caractères à moindre progrès présentent une moindre diminution de variabilité dans $P A_{1}$. Afin d'évaluer simultanément les 2 volets (moyenne et variabilité), il est possible de confronter les performances moyennes et extrêmes de $\mathrm{PA}_{0}$ et $\mathrm{PA}_{1}$ (tableau $\mathrm{VI}$ ), révélatrices d'une certaine variabilité résiduelle après 2 cycles. Nous confirmons alors l'effet restrictif du cycle de sélection récurrente sur la variation globale avec, pour la majorité des caractères, les relations suivantes :

$\operatorname{Max} P A_{0}>M_{a x} P A_{1}$

Min $\mathrm{PA}_{0}<\operatorname{Min} \mathrm{PA}_{1}$

\section{Variances génétiques pour $P A_{1}$}

Compte tenu de l'insuffisance des variances phénotypiques pour caractériser la variation existante, nous examinons ici les variances génétiques, seulement calculées pour $P A_{1}$.

Afin de pouvoir comparer les différents caractères entre eux, l'indice $S^{2} G / \bar{x}$ (rapport de la variance à la moyenne du caractère) servira de référence. Sur l'examen de ce rapport (tableau VII), nous retiendrons la comparaison pour la génération $\mathrm{PA}_{1} \mathrm{~S}_{1}$ révélant 3 caractères à indice fort $\left(R B, H A, N G_{3} E\right)$ parmi un ensemble de caractères à indice faible à l'issue du cycle $\mathrm{PA}_{1}$.

\section{Discussion sur l'évolution des variances au cours des 2 cycles dans PA}

\section{Variances phénotypiques globales}

La diminution de variance forte pour les caractères sélectionnés efficacement est liée pour la 


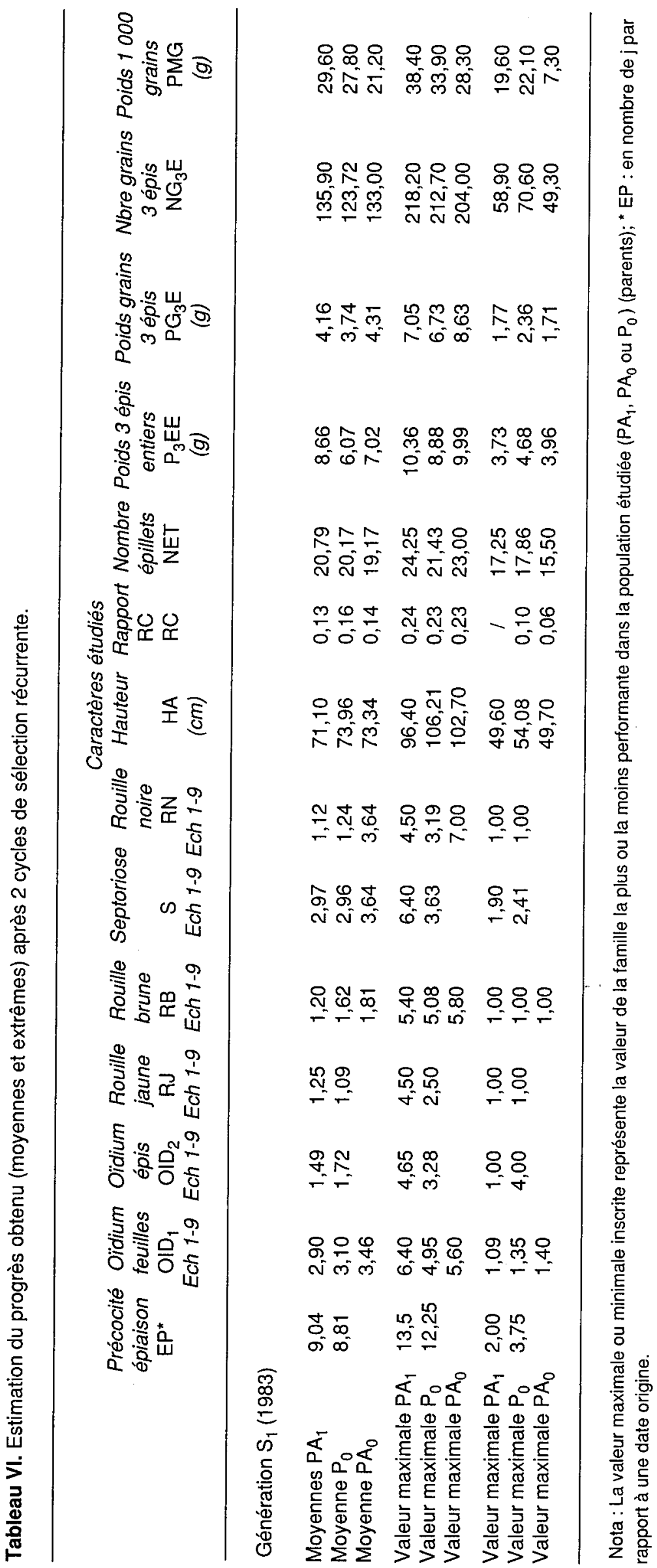




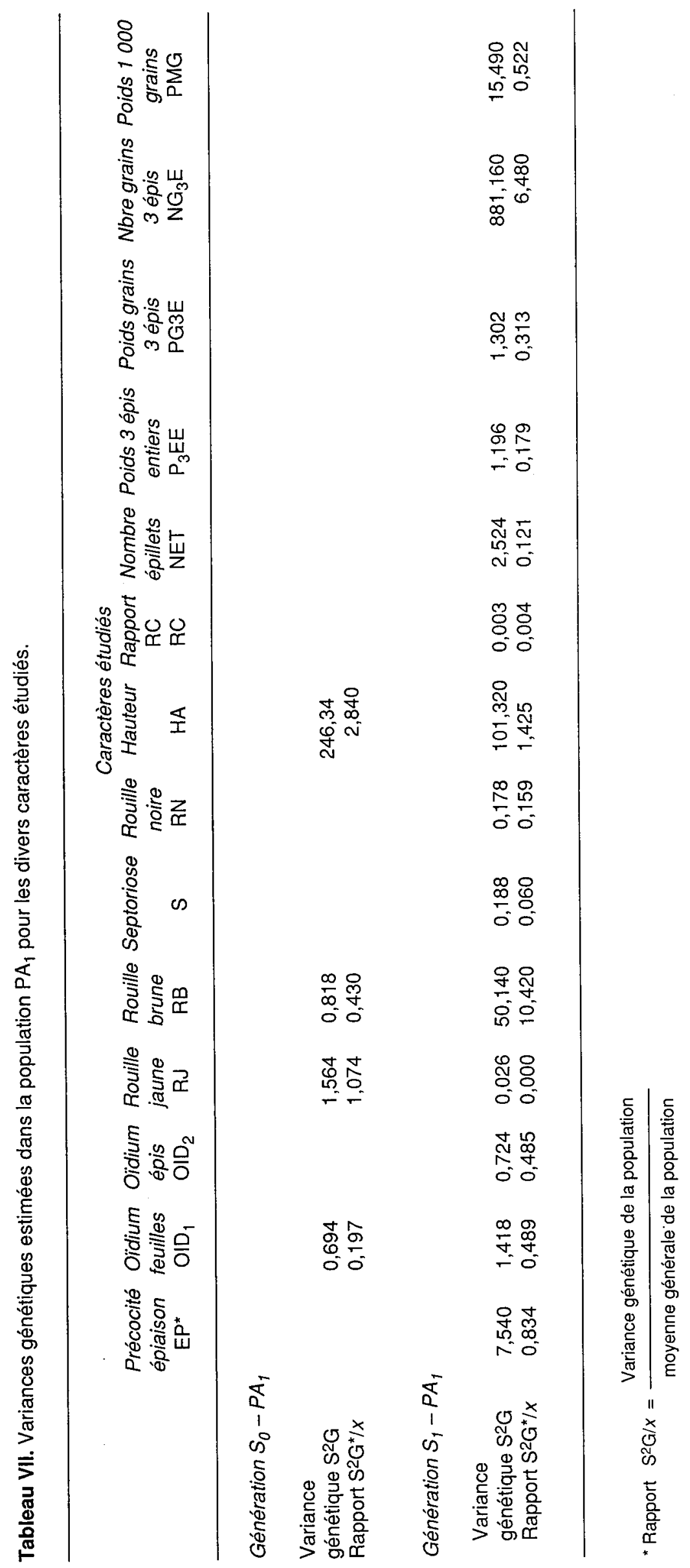


plupart des cas à une sélection sur quelques gènes (gènes récessifs de résistance pour $R B$, $R J, R N$, élimination des extrêmes hauts et courts pour HA). Bien entendu, on peut penser que la sélection a retenu l'ensemble des génotypes intéressants mais ceci sous-entend une difficulté de progression pour les cycles ultérieurs. En effet, la sélection s'est ici faite sur des extrêmes à partir de gènes majeurs sans apprécier l'ensemble du fond génétique de gènes mineurs (pour le cas des rouilles, fond génétique de résistance intéressant en cas de modifications des races; Lewellen et al, 1967). Cette deuxième sélection, sur le fond génétique, intéressante dans une optique d'amélioration à long terme, peut avoir été hypothéquée dans ces premiers cycles, efficaces certes, mais sans doute brutaux.

Pour tous les autres caractères (à hérédité quantitative pour la plupart), la moindre diminution de variance permet d'envisager des possibilités d'amélioration ultérieure.

Cette évolution des variances phénotypiques observées est toutefois à nuancer du fait qu'elle comprend non seulement l'évolution vraie de la variance de chaque caractère mais aussi la variance environnementale et sa fluctuation (modes de notation pour les parasites, effets du milieu). Par ailleurs, la comparaison avec la variance $P A_{0}$ concerne un échantillon restreint de cette population initiale, de moindre précision.

Dans ce sens, mais avec des dispositifs expérimentaux plus élaborés, l'estimation des variances génotypiques est, de beaucoup, plus satisfaisante pour cette analyse.

\section{Variances génétiques dans $P A_{1}$}

Cet examen permet d'analyser l'évolution intracycle montrant une fluctuation de l'indice $S^{2} g / \bar{x}$ entre $S_{0}$ et $S_{1}$ avec 2 caractères pour lesquels on note une diminution ( $R J, H A$ ) et 2 caractères pour lesquels on note une augmentation $\left(O I D_{1}\right.$, $R B)$. Ce dernier cas correspond en fait à une diminution brutale de la moyenne de ces caractères (sélection efficace) augmentant ainsi numériquement l'indice.

Par ailleurs, compte tenu de l'éventail de caractères impliqués, nous n'examinerons que la génération $\mathrm{PA}_{1} / \mathrm{S}_{1}$ pour la comparaison des indices intragénération. Les faibles indices observés $\left(S^{2} G / \bar{x}\right)$ montrent une variation résiduelle faible pour les caractères correspondant à une sélection sévère et efficace (héritabilité moyenne -forte).
Par contre, les caractères conservant un indice fort correspondent à des critères qui ont été étudiés mais ont subi une faible pression de sélection (hormis le caractère $R B$ pour lequel la variabilité résiduelle importante est liée à l'exis. tence de plusieurs systèmes de résistance superposés).

Ces résultats montrent l'importance de ne pratiquer qu'une pression de sélection modérée du fait d'un objectif d'amélioration à long terme en sélection récurrente et du fait d'une poursuite du programme récurrent par un schéma de création variétale pour lequel une variabilité initiale est nécessaire. D'autre part, cette restriction de la variabilité confirme la nécessité de rebrassage par intercroisements afin de restaurer une certaine variation. Ces intercroisements, pour l'espèce et les caractères concernés se confirment être indispensables dès la deuxième génération de chaque cycle, obligeant à rejeter ici l'éventualité d'une troisième génération de sélection avant intercroisement. Si la sélection pouvait être efficace dès la première génération pour l'ensemble des caractères, il serait même judicieux d'envisager l'alternance complète : 1 génération de sélection $/ 1$ génération d'intercroisements.

\section{DISCUSSION ET CONCLUSION GÉNÉRALES}

Le bilan de ces 2 cycles de sélection récurrente sur la population PA montre l'obtention d'un certain progrès. Quoique partiellement mesuré (seulement les 2 premiers cycles), le progrès réalisé est marqué essentiellement par l'évolution positive du niveau moyen des caractères retenus en sélection. Ceux-ci, intégrés dans les index utilisés, évoluent en majorité positivement avec un progrès d'autant plus grand que l'héritabilité est forte (et donc la sélection pratiquée efficace).

Par ailleurs, la variation après 2 cycles a stagné ou régressé, témoignant de l'impérative nécessité de pratiquer une faible pression de sélection à chaque cycle et de poursuivre l'accumulation des cycles d'intercroisement. Ce bilan montre, toutefois, par rapport aux travaux antérieurs réalisés sur 1 ou quelques critères, qu'un schéma de sélection récurrente multicaractères peut s'avérer efficace.

Notamment pour les caractères à hérédité complexe, 2 cycles (et donc 1 intercroisement) sont insuffisants pour sélectionner les génotypes 
performants et pour juger complètement d'un tel schéma. Les quelques travaux antérieurs ont plutôt montré que 5 cycles représentaient un chiffre moyen nécessaire lorsqu'on améliore des caractères quantitatifs (Jensen, 1978; Gupton, 1981; Busch et Kofoid, 1982; Weber, 1986).

Nous avons proposé ici plusieurs modalités d'évaluation de ce schéma. lls concordent correctement mais sont tous basés sur la comparaison de performances en populations. En effet, compte tenu de la difficulté d'obtention rapide de lignées, l'évaluation en lignées n'a pu être réalisée bien qu'étant le mode d'évaluation idéal puisque proche de la finalité du schéma (création variétale de lignées). Ce mode d'évaluation déjà employé pour quelques caractères en sélection récurrente (Busch et Kofoid, 1982; Loffler, 1983) pourrait permettre de comparer un nombre égal de lignées tirées de $P A_{0}$ et $P A_{1}$ (et à l'avenir de $\mathrm{PA}_{i}$ ). La production de ces lignées serait susceptible d'être réalisée en générations accélérées (SSD ou haplodiploïdisation si cette méthode est applicable sans biais génotypique dans l'espèce).

Dans cette espèce autogame où la sélection récurrente est peu employée mais semble tout à fait séduisante, compte tenu des inconvénients d'utilisation du schéma variétal classique, nous montrons que la plupart des caractères analysés sont améliorés par le schéma retenu malgré leur diversité et celle de leur déterminisme. Cette étude s'est toutefois limitée à une seule population artificiellement créée et à une seule méthode récurrente. II conviendrait notamment d'étudier avec la même population, divers processus d'intercroisements : processus disruptif (croisement des meilleures unités avec les moins bonnes; croisement d'unités retenues sur un index partiel avec d'autres unités retenues sur un autre index partiel). De même, après avoir montré tout l'intérêt du recroisement systématique pour le maintien de la variabilité de la population, diverses modalités de réalisation des croisements permettraient d'élargir le champ des possibilités de recombinaison notamment par l'utilisation d'un système de stérilité mâle facili- tant le brassage plus exhaustif à l'intérieur de la population. L'ensemble de ces perspectives pourrait être envisagé dans le cadre d'une étude méthodologique comparative des diverses options proposées.

\section{RÉFÉRENCES}

Bachacou J, Masson JP, Millier C (1981) Manuel de la programmation statistique Amance 1981. INRA (Dept Biométrie), $516 \mathrm{p}$

Busch RH, Kofoid K (1982) Recurrent selection for kernel weight in spring wheat. Crop Sci 22, 568-572

Doggett H, Eberhart SA (1968) Recurrent selection in sorghum. Crop Sci 8, 119-121

East EM, Jones DF (1920) Inbreeding and outbreeding (Lipincott JB) Philadelphia

Frey KJ (1981) Plant breeding II. lowa Univ Press, $497 p$

Gallais A (1977) Amélioration des populations, méthodes de sélection et création de variétés. I. Synthèse sur les problèmes généraux et sur les bases théoriques pour la sélection récurrente intrapopulation. Ann Amelior Plant 27, 281-329

Gupton CL (1981) Phenotypic recurrent selection for increased leaf weight and decreased alakaloïd content of barley tobacco. Crop Sci 21, 921-925

Hayes J, Garber M (1919) Synthetic production of high protein corn in relation to breeding. $J$ Am Soc Agron $11,309-318$

Jensen NF (1978) Composite breeding methods and the DSM system in cereals. Crop Sci 18, 622-626

Lewellen RT, Sharp EL, Hehn EK (1967) Major and minor genes in wheat for resistance to Puccinia striiformis and their responses to temperature changes. Can J Bot 45, 2155-2172

Loffler CM (1983) Recurrent selection for grain protein percentage in hard red spring wheat. Crop Sci 23, 1097-1101

Thomas G, Doussinault G, Trottet M (1991) Méthodologie de l'amélioration du blé tendre. I. Création par croisements et analyse d'une population artificielle à 16 parents, base de cette étude méthodologique. Agronomie 11, 359-368

Weber WE (1986) The efficiency of repeated crosses within a population of self fertilizing species. $Z$ Pflanzenzuecht 91, 129-139 